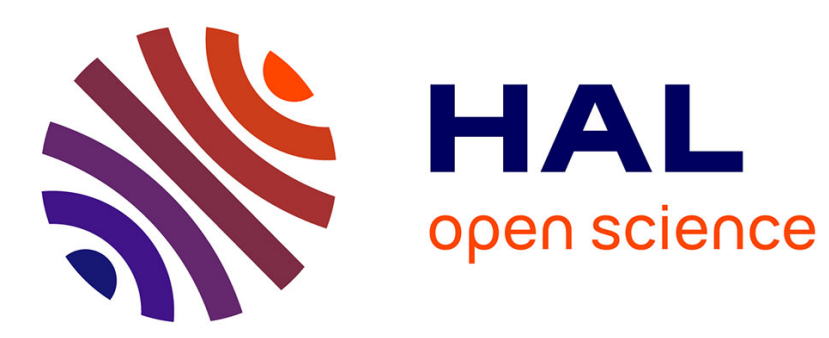

\title{
EXPÉRIENCES BASÉES SUR LES INÉGALITÉS DE BELL
}

\author{
Alain Aspect
}

\section{To cite this version:}

Alain Aspect. EXPÉRIENCES BASÉES SUR LES INÉGALITÉS DE BELL. Journal de Physique Colloques, 1981, 42 (C2), pp.C2-63-C2-80. 10.1051/jphyscol:1981203 . jpa-00220689

\section{HAL Id: jpa-00220689 https://hal.science/jpa-00220689}

Submitted on 1 Jan 1981

HAL is a multi-disciplinary open access archive for the deposit and dissemination of scientific research documents, whether they are published or not. The documents may come from teaching and research institutions in France or abroad, or from public or private research centers.
L'archive ouverte pluridisciplinaire HAL, est destinée au dépôt et à la diffusion de documents scientifiques de niveau recherche, publiés ou non, émanant des établissements d'enseignement et de recherche français ou étrangers, des laboratoires publics ou privés. 
JOURNAL DE PHYSIQUE

Colloque C2, supplément au $n^{\circ} 3$, Tome 42 , mars 1981

page $\mathrm{C} 2-63$

EXPÉRIENCES BASÉES SUR LES INÉGALITÉS DE BELL

A. Aspect

Institut d'optique, Université Paris-Sud, B.P. 43, 91406 Oxsay Cedex, France ou

Ecole Normale Supérieure de l'Enseignement Technique 94230 Cachan, France

Résumé.- On expose la démarche conduisant de l'expérience de pensée à une expérience réelle dans le cas des photons visibles, en faisant apparaître les hypothèses supplémentaires nécessaires. Après une reviue des expériences rêalisées à ce jour, on présente une expérience en cours, utilisant des analyseurs variabjes dans le temps.

Abstract.- One presents the paths from a thought experiment to a real experiment with visible photons, pointing out the necessary supplementary assumptions. After a survey of the experiments already performed, a timing experiment (with variable analysers), now in progress, is described.

On va s'efforcer de montrex le cheminement conduisant des "expériences de pensée" ("Gedankenexperiment") aux expériences réelles, afin de savoir quelles conclusions tirer de ces expériences réelles. On essaiera de présenter les choses de façon à ce qu'elles soient compréhensibles par les non-scientifiques, qui devraient pouvoir sauter tous les passages "manifestement pour initiés", tout en suivant les grandes lignes du raisonnement.

On n'a donné aucune bibliographie; le lecteur désirant plus de dêtails trouvera les références bibliographiques dans le très complet article de revue de J.F. Clauser et A. Shimony publié à Reports on Progress in Physics (Rep. Prog. Phys. 1978, 41, 1831-1927).

1. Présentation d'une situation expérimentale réalisable en principe utilisant des photons de basse énergie (visibles).- 1.1.Rappel de I'expérience de pensée.- 1.1.1.Schéma expérimental (fig.1).-

Une source émet une paire de particules $\left(v_{A}, v_{B}\right)$ (par exemple deux spins 1/2), ce qui déclenche un "détecteur d'émission". I est un appa-reil de mesure donnant un résultat dichotomique ( $I_{+}$ou $I_{-}$) lorsque $v_{A}$ $y$ entre (par exemple, un filtre Stern et Gerlach); on peut choisir à volonté un paramètre de réglage a de l'appareil I (par exemple a est l'orientation du Stern et Gerlach).

L'appareil II agit de façon analogue sur $\nu_{B}$ (résultat II+ ou II_' paramètre b). 


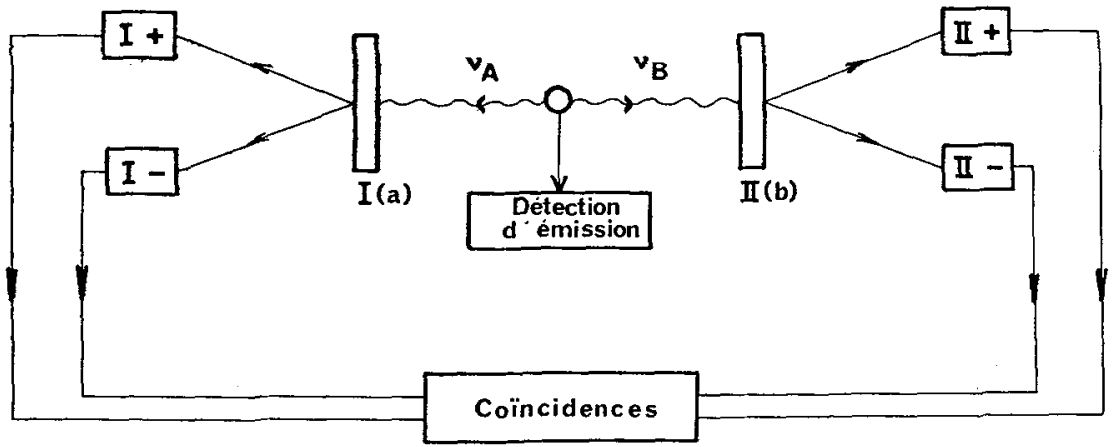

Fig. 1.- Expérience de pensée.

Les particules $\nu_{A}$ et $\nu_{B}$ sont soumises à des mesures dichotomiques. Les appareils de mesure I et II possèdent chacun un paramètre réglable $a$ et $b$. On peut mesurer les corrélations entre les résultats de mesure sur $\nu_{A}$ et $\operatorname{sur} \nu_{B}$.

On peut, grâce à un dêtecteur de coïncidences, mesurer la probabilité d'obtenir les résultats $I_{+}$pour $v_{A}$ et II + pour $v_{B}$ (les paramètres valant $a$ et $b$ ) :

$$
\mathrm{P}_{++}(\mathrm{a}, \mathrm{b})=\frac{\text { Nombre de détections en coïncidence en } \mathrm{I}_{+} \text {et } \mathrm{II}}{\text { Nombre de paires émises }}
$$

On peut de même, et simultanément, mesurer les probabilités $P_{+-}(a, b), P_{-+}(a, b)$ et $P_{--}(a, b)$.

Il est alors possible, pour un couple donné, de mesurer au cours d'une seule expérience la quantité $E(a, b)$ :

$$
E(a, b)=P_{++}(a, b)-P_{+-}(a, b)-P_{+-}(a, b)+P_{--}(a, b)
$$

(que I'on peut interpréter comme valeur moyenne de $\mathrm{A} \times \mathrm{B}, \mathrm{A}$ valant \pm 1 et $B= \pm I$ suivant les résultats de mesure en $I$ et II).

1.1.2. Inégalités de Bell (généralisées par clauser, Horne, Holt, shimony).- S'il existe des situations où les mesures faites sur $v_{A}$ et $v_{B}$ sont corrélées (ce qui apparât sur les probabilités de détection en coïncidence), on peut être tenté d'interpréter ces corrélations par des causes situées dans le passé commun de $\nu_{A}$ et $\nu_{B}$, puisque $\nu_{a}$ et $\nu_{B}$ proviennent de la même source.

En admettant un tel schéma explicatif, auquel on ajoute une hypothèse de localité, on démontre les inégalités de Bell, que l'on peut donner sous la forme suivante. Soit la quantité :

$$
S=E\left(a_{1}, b_{1}\right)-E\left(a_{1}, b_{2}\right)+E\left(a_{2}, b_{1}\right)+E\left(a_{2}, b_{2}\right)
$$


formée à partir de 4 mesures successives, correspondant à 4 couples d'orientations pour les appareils de mesure; l'inégalité B.C.H.H.S. s'écrit alors:

$$
-2 \leq S \leq 2
$$

1.1.3. Conflit avec la mécarique quantique.- Il existe des situations (rares) où les prédictions de la mécanique quantique violent les inégalités B.C.H.H.S. Dans l'exemple cité au paragraphe 1.1.1., si les deux spins $I / 2$ sont dans l'état singulet, un calcul élémentaire de mécanique quantique conduit à:

$$
E(a, b)=-\cos (a, b)
$$

Dans la situation:

$$
\left(a_{1}, b_{1}\right)=\left(b_{1}, a_{2}\right)=\left(a_{2}, b_{2}\right)=3 \pi / 4
$$

(et donc $\left.\left(a_{1}, b_{2}\right)=3 \pi / 4\right)$, il est facile de vérifier que la prédiction de la M.Q. est : $S=-2 \sqrt{2} \simeq-2,8$ en contradiction evidente avec I'inêgalité $S \geq-2$

1.I.4 On peut résumer la situation par le schéma :

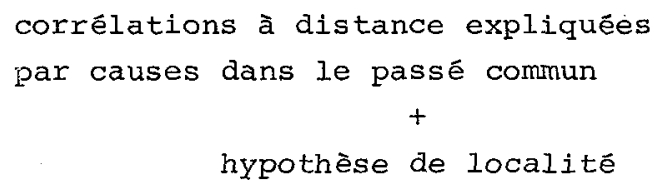

Or, il existe des situations oũ la M.Q. prévoit des corrélations assez fortes pour violer les inégalités de Bell. La M.Q. ne peut donc pas se réduire au schêma explicatif conduisant aux inégalités de Bell. Il y a un conflit, portant sur des prédictions de résultats de mesure. I'expérience est donc, en principe, capable de rêsoudre cé conflit. Remarque 1.- La mécanique quantique ne joue aucun rôle dans 1'élaboration des inégalités de Bell; elle est utilisée pour trouver des situations expérimentales où il y a de fortes corrélations et où elle prévoit une violation des inégalités de Bell.

Remarque 2.- De nombreuses situations où l'on observe des corrélations entre mesures à distance obêissent aux inégalités de Bell; ce sont, par exemple, les cas où l'évolution des systèmes est décrite complètement par la mécanique classique, ou bien par la théorie classique des champs. 1.2. Possibilité̃ d'expériences "sensibles"_dvec_des_photons_de_basse énergie.- 1.2.1. Mesures de polarisation de photons.- De la lumière tombant sur un polariseur birêfingent émerge, suivant sa polarisation, 
soit dans la direction +, soit dans la direction -. On détecte la lumière émergeant dans la direction + ou - par un système à comptage de photons (photomultiplicateur). On peut dire qu'on effectue une mesure dichotomique sur les photons; l'appareil de mesure possède un paramètre variable (son orientation a). On a donc une situation analogue à celle des spins $1 / 2$ arrivant sur un Stern et Gerlach.

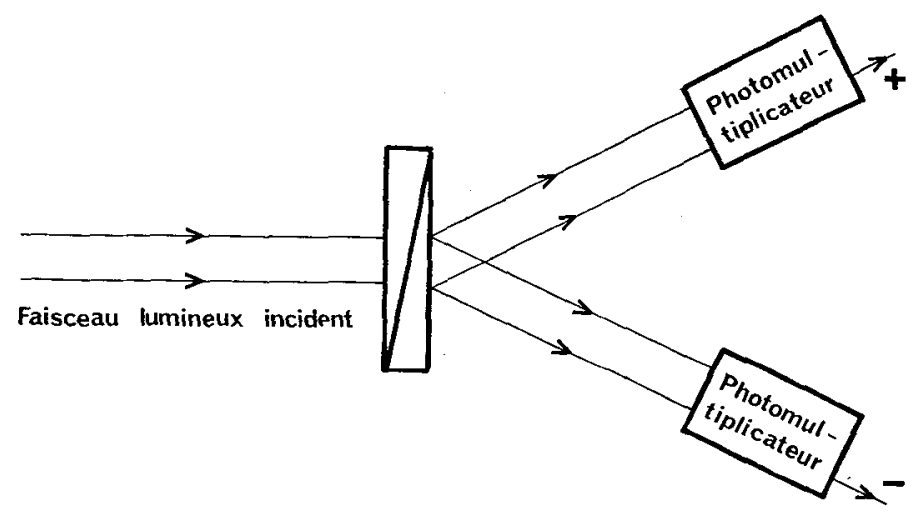

Fig.2.- L'analyse d'un faisceau de lumière par un polariseur biréfringent, suivi par deux photomultiplicateurs, constitue une mesure "dichotomique" d'un fiux de photons. On peut faire tourner l'ensemble polariseur + détecteurs autour de 1 'axe du faisceau incident (paramètre réglable de l'appareil de mesure).

1.2.2. Emission de paires fortement corrélées.- Soit une cascade radiative atomique $J=0 \rightarrow J=I \rightarrow J=0$. ( $J=$ moment cinétique atomique) au cours de laquelle un atome émet successivement deux photons $v_{A}$ et $\nu_{B}$.

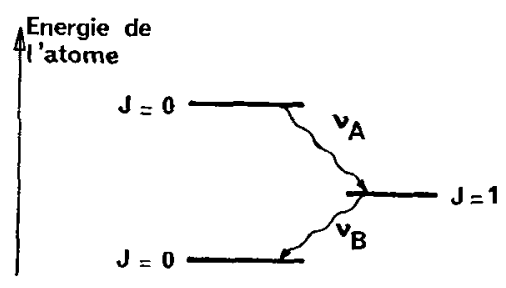

Fig.3.- Cascade radiative atomique $\mathrm{J}=0 \rightarrow \mathrm{J}=\mathrm{I} \rightarrow \mathrm{J}=0$. Dans une cascade radiative telle que celleci, l'atome émet une paire de photons. Les corrélations prévues pour les mesures de polarisation de ces photons sont analogues aux corrélations prêvues au $\S 1.1$. avec les spins $1 / 2$ dans l'état singulet.

Il est facile de montrer que:

$$
\left.\left|\nu_{A}, \nu_{B}\right\rangle=\frac{1}{\sqrt{2}}\left\{\left|+_{A},+_{B}>-\right|--_{A^{\prime}}-{ }_{B}\right\rangle\right\}
$$

oũ $\mid+>$ et $\mid->$ sont deux états orthogonaux de polarisation linéaire.

On peut alors utiliser une telie source de paires de photons dans une expérience du type 1.1.1. (Fig.l), où les appareils de mesure sont 
des polariseurs biréfringents. Un calcul élémentaire de M.Q. donne:

$$
\begin{aligned}
& P_{++}(a, b)=\frac{1}{2} \cos ^{2}(a, b) \\
& P_{+-}(a, b)=\frac{1}{2} \sin ^{2}(a, b)
\end{aligned}
$$

Pour les orientations particulières suivantes (Fig.4):

$$
\begin{aligned}
& \left(a_{1}, b_{1}\right)=\left(b_{1}, a_{2}\right)=\left(a_{2}, b_{2}\right)=\frac{\pi}{8} \\
& \left(a_{1}, b_{2}\right)=3 \frac{\pi}{8}
\end{aligned}
$$

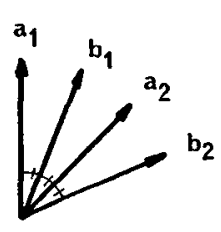

Fig.4.- Orientations des polariseurs conduisant à la violation maximale des inégalités de Bell.

Le calcul quantique conduit à :

$$
S=2 \sqrt{2} \simeq 2,8
$$

Cette prédiction est en contradiction évidente avec l'inégalité (3): $S \leq 2$. En résumé, on a présenté une situation reproduisant "l'expérience idéale" dans laquellé la mécanique quantique prédit une violation des inégalités de Bell, et utilisant des systèmes existant.

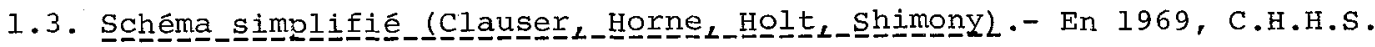
ont proposé la première expérience techniquement réalisable, utilisant des photons visibles. Leur schéma est plus simple que le précédent car, d'une part il ne nécessite pas de détecteur d'émission, et d'autre part on n'utilise qu'une "voie" de mesure de chaque polariseur. (la voie t, par exemple) (Fig.5).

Dans cette expérience, on mesure le nombre de coïncidences $N_{++}(a, b)$ et le nombre d'évènements simples détectés de chaque côtê $\mathrm{N}_{+}(a)$ et $\mathrm{N}_{+}(b)$. on fait le lien avec le schéma précédent en attribuant au résultat la signification "non-détection", et en remarquant que:

$$
\mathrm{N}_{+}(\mathrm{a})=\mathrm{N}_{++}(\mathrm{a}, \mathrm{b})+\mathrm{N}_{+-}(\mathrm{a}, \mathrm{b})
$$

et de même pour $\mathrm{N}_{+}(\mathrm{b})$ (ceci n'étant vrai que dans le cas idéal). on peut alors former une quantité prenant en compte directement les résultats des comptages pour quatre couples d'orientations:

$S^{\prime}=N_{++}\left(a_{1}, b_{1}\right)-N_{++}\left(a_{1}, b_{2}\right)+N_{++}\left(a_{2}, b_{1}\right)+N_{++}\left(a_{2}, b_{2}\right)-N_{+}\left(a_{2}\right)-N_{+}\left(b_{1}\right)$ 
et l'inêgalité B.C.H.H.S. s'écrit (après des transformations algébriçues n'offrant aucune difficulté):

$$
S^{\prime} \leq O
$$

Pour les orientations particulières vues en 1.2.2. (Fig.4), la mécanique quantique prédit:

$$
S^{\prime}=(\sqrt{2}-1) \cdot N \simeq 0,4 \cdot N
$$

(N étant le nombre total de paires prises en compte), ce qui est en conflit évident avec l'inégalité B.C.H.H.S. (IO) : $S^{\prime}<0$.

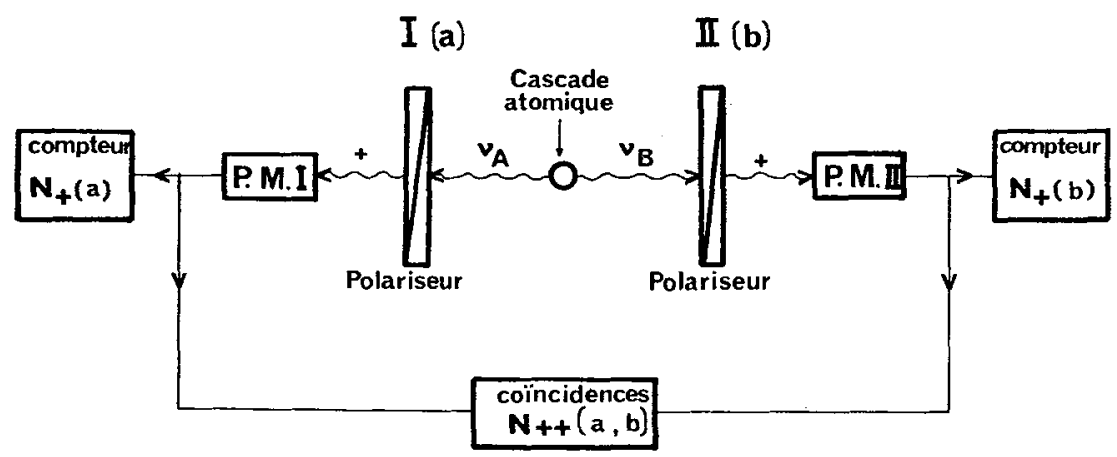

Fig.5.- Schéma pratique simplifié (Clauser, Horne, Holt, Shimony). On ne fait de mesure que sur une des deux voies de sortie de chaque polariseur, et on fait à la fois des comptages en coïncidence et des comptages simples. On aboutit encore à un conflit entre des inégalités de Bell généralisées et les prédictions de la M.Q. (§ 1.3$)$.

Conclusion de la partie 1.- On est passé d'un schéma théorique irréalisable, bien que concevable en principe (expérience de pensée), à un schéma expérimental réalisable en principe avec des systèmes existant. Ce schêma expérimental constitue un test "sensible", puisqu'il existe des situations pour lesquelies la mécanique quantique prévoit que les quantités mesurées violent les inégalités de Bell.

2. Les expériences effectivement réalisées.- 2.1 Nécessittê_d'hypothèses supplêmentairres.-2.1.1. Imperfections à prendre en compte.-

- Mauvais rendement des détecteurs: un photon tombant sur un photomultiplicateur a une probabilité de l'ordre de 10\% d'être détecté.

- Mauvaise corrélation angulaire: dans une cascade atomique, les deux photons $\nu_{A}$ et $\nu_{B}$ n'ont aucune raison de partir dans des directions opposées. On essaie de les collecter au mieux, mais néanmoins, chaque fois qu'un photon. $\nu_{A}$ entre dans le polariseur $I$, il $y$ a moins de $10 \%$ de chances que $\nu_{B}$ entre dans II. 
- Absorption par les divers éléments optiques: elle donne lieu à quelques pertes supplémentaires.

2.1.2. Absence de confilt.- Compte-tenu de ces defauts, la mécanique quantique prévoit des taux de comptage en coincidence 100 fois plus faibles que les taux de comptage simples, et 11 est alors évident que S' (éq. 10 a) sera toujours nêgatif.

Dans ces conditions, il n'y a pas de conflit entre les prédictions de la mêcanique quantique et les inégalitês de Bell.

2.1.3. Hypothèse supplémentaire C.H.H.S.- Il est raisonnable d'admettre que la probabilité qu'un photomultiplicateur détecte un photon est indépendante:

- de l'orientation du polariseur placé devant ce photomultiplicateur(ou de son éventuelle absence) ;

- de "l'histoire passeee du photon" (cette histoire qui, dans le formalisme des variables cachées est la cause des corrélations observées).

On montre alors qu'on peut établir des inégalités analogues aux précédentes, mais où les comptages simples $\mathrm{N}_{+}(\mathrm{a})$ et $\mathrm{N}_{+}(\mathrm{b})$ sont remplacés par des comptages en coincidence $N_{++}(a, \infty)$ et $N_{++}(\infty, b)$ (le symbole $\infty$ signifie que le polariseur correspondant a été enlevé. La relation (9), qui n'est plus valable dans le cas rêel, peut alors être remplacée par:

$$
\mathrm{N}_{++}(\mathrm{a}, \infty)=\mathrm{N}_{++}(\mathrm{a}, \mathrm{b})+\mathrm{N}_{+-}(\mathrm{a}, \mathrm{b})
$$

L'inégalité B.C.H.H.S. S'écrit maintenant:

$$
S^{\prime \prime} \leq \mathrm{O}
$$

avec

$S^{\prime \prime}=N_{++}\left(a_{1}, b_{1}\right)-N_{++}\left(a_{1}, b_{2}\right)+N_{++}\left(a_{2}, b_{1}\right)+N_{++}\left(a_{2}, b_{2}\right)$

Dans le cas réel, et pour les orientations particulières de la figure 4, la mécanique quantique prévoit alors la même violation que dans le cas idéal ( $(1.3)$ :

$$
S^{\prime \prime}=(\sqrt{2}-1) \cdot N \simeq 0,4 \cdot N
$$

(où $N$, nombre total de paires qui seraient détectées en enlevant les deux polariseurs, est manifestement positif) . Ceci est en conflit avec (12 a).

2.1.4. Hypothèse de Clauser et Horne.- On peut utiliser une autre hypothèse, qui semble encore plus faible: la probabilité de détection par un photomultiplicateur, pour un photon donné, n'augmente pas lorsqu'on ajoute un polariseur devant le photomultiplicateur. I1 n'est pas sûr que cette hypothèse soit réellement plus faible (existencé de contreexemples, avec des photomultiplicateurs sensibles à la polarisation) . 
2.1.5. Statut de ces hypothèses supplémentaires.- Ces hypothèses apparaissent très raisonnables à la lumière de la physique connue. Elles ne sont nëanmoins susceptibles d'aucune vérification expérimentale directe, car elles énoncent des propriétés relatives au comportement d'un photomultiplicateur pour chaque photon. Or, lorsque l'on envisage le formalisme des inégalités de Bell, on accepte de considérer que des photons que nous croyions identiques sont en fait porteurs de propriétés distinctes (ce sont ces propriêtés - "variables cachées" - qui sont censées expliquer les corrélations observées). Il y aurait donc une inconséquence logique à tenter de vérifier une telle propriété en envoyant sur un photomultiplicateur un flux de photons que nous croirions tous identiques, et en faisant des statistiques.

La seule façon d'éliminer ce genre d'hypothèse serait d'avoir des systèmes parfaits, avec des probabilités de détection de 100\% manifestement indépendantes de tout paramètre connu ou inconnu, ce qui conduirait à une violation directe des inégalités vues en 1.3.

Conclusion de la partie 2.1.- Les expériences réalisées sur des photons visibles ne seront en fait des expëriences "sensibles" que si l'on accepte une de ces hypothèses supplémentaires.

2.2._L'expérience_de_Pry_et_Thomoson_(1976)-- on a choisi de aécrire cette expérience pour montrer la finesse des techniques en jeu.

2.2 .1 . La source.-

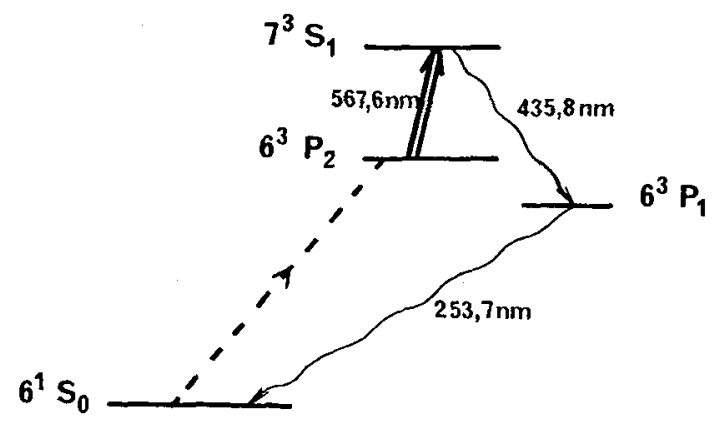

Fia.6.- Cascade radiative dans le mercure utilisée par Fry et Thompson. L'excitation des atomes faite par bombardement électronique vers le niveau $6^{3} \mathrm{P}_{2}$ suivi d'une excitation sélective par laser vers le niveau $7^{3} \mathrm{~S}_{1}$ des "bons" iotopes.

F. et $T$. ont utilisé une cascade dans le mercure dont la mécanique quantique prévoit qu'elle est un bon candidat. On a un jet atomique de mercure, ce qui permet d'avoir des atomes "libres", dont le comportement est facile à décrire par la théorie. Un bombardement électronique excite les atomes, certains dans le niveau $6{ }^{3} \mathrm{P}_{2}$ (Fig.6). Un laser éclaire ces atomes excités; sa longueur d'onde correspond à la transition $6{ }^{3} \mathrm{p}_{2} \rightarrow$ 
$7^{3} \mathrm{~S}_{1}$, ce qui permet finalement d'obtenir des atomes dans 1 'état $7^{3} \mathrm{~S}_{1}$ dont certains se désexciteront en réémettant deux photons de longueurs d'onde $4358 \AA$ et $2537 \AA$, sur lesquels porteront les mesures $\left({ }^{1}\right)$. Deux filtres à ces deux longueurs d'onde sélectionnent chacun de ces deux photons à l'entrée des voies de mesure.

Un "detail" donne une idée de la nécessité de tout contrôler: on ne prêvoit une violation de l'inégalité de Bell que pour certains isotopes (pairs) du mercure. On met à profit les très petites différences de longueurs d'onde correspondant à des transitions identiques dans les différents isotopes pour n'exciter, avec le laser $5676 \AA$, que l'isotope $\mathrm{Hg}^{200}$ constituant un bon candidat.

2.2.2. La détection.- Après passage dans les polariseurs, les photons tombent sur les photomultiplicateurs PMI et PM2. A la sortie de ces photomultiplicateurs, des impulsions indiquentqu'une détection a eu lieu.
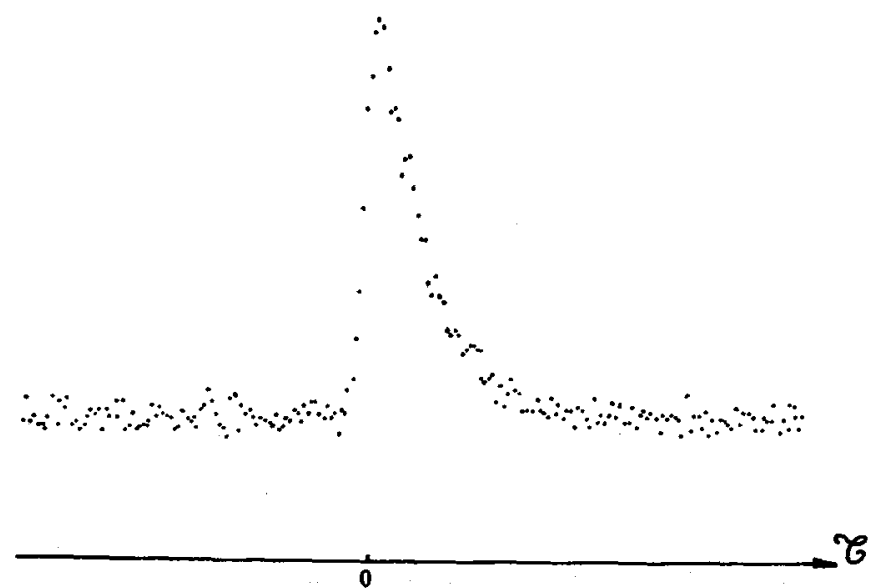

Fig.7.- Exemple de signal de corrélation obtenu en étudiant le nombre de coïncidences observees en fonction du retard $\tau$. Le pic autour de $\tau=0$ correspond aux col̈ncidences "vraies" photons émis par le même atome. Le fond plat correspond à des colncidences "fortuites". La décroissance exponentielle du pic correspond à l'existence d'une "durée de vie" du niveau intermédiaire de la cascade.

Lorsque deux impulsions sont comptées simultanément en PMI et PM2, on dêtecte une coüncidence. Mais, de nombreux atomes ayant été

\footnotetext{
( ${ }^{1}$ La cascade utilisée ici est du type $J=1 \rightarrow J=1 \rightarrow J=0$, mais, dans ce cas, cela ne change rien aux raisonnements faits sur les cascades $\mathrm{J}=\mathrm{O} \rightarrow \mathrm{J}=\mathrm{I} \rightarrow \mathrm{J}=0$.
} 
excités simultanément, comment savoir si cette coïncidence est bien due à deux photons $v_{A}$ et $v_{B}$ provenant du même atome (c'est sur de telles paires que porte le test) et non à des photons $\nu_{A}$ et $\nu_{B}$ provenant d'atomes différents? On peut discriminer ces situations en mesurant le retard entre les détections en I et II, et en étudiant la répartition des "coincidences" en fonction de ce retard.

On observe alors un diagramme comportant un "fond" de hauteur constante au-dessus duquel se détache un "pic" commençant au retard nul (Fig.7).

On interprète le fond comme dû à des cö̈ncidences "fortuites" correspondant ã des photons émis par des atomes distincts (car, dans ce cas, aucun retara n'est privilégié). Au contraire, le pic correspona à deux photons êmis dans la même cascade du même atome: Iorsque $\nu_{A}$ a été émis, $\nu_{B}$ est émis dans les instants qui suivent, avec une loi de probabilité correspondant au pic observé.

On comptera donc comme signal les coïncidences du pic. Il est remarquable de noter que ces techniques permettent d'obtenir un excellent "rapport signal sur bruit" en dépit de circonstances apparamment très défavorables au départ (moins d'une colncidence pour plus de cent coups à chacun des photomultiplicateurs).

2.2.3. Résultats.- La façon la plus commode d'exploiter les résultats consiste à former une quantité sans dimensions $\Delta$ à partir des taux de comptages. L'inégalitê de Bell (compte-tenu de l'hypothèse supplémentaire) s'ecrit alors:

$$
\Delta \leq 0,25
$$

tandis que la mécanique quantique prévoit une violation maximale:

$$
\Delta_{\text {M.Q. }}=\frac{\sqrt{2}}{4} \simeq 0,35 \text { (cas idéal) }
$$

avec les orientations de polariseurs indiquées en 1.2.2. (Fig.4). Le résultat expérimental de Fry et Thompson est :

$$
\Delta_{\text {exp }}=0,296 \pm 0,014
$$

ce qui viole clairement l'inégalité (13). Compte-tenu de quelques imperfections du montage, la prédiction de la mécanique quantique est :

$$
\Delta_{\text {M.Q. }}=0,294 \pm 0,007 \text { (cas réel) }
$$

L'expérience de Fry et Thompson est en parfait accord avec la mécanique quantique et viole les inégalités B.C.H.H.S. utilisant l'hypothèse supplémentaire sur les photomultiplicateurs $\left({ }^{2}\right)$.

(2) Note ajoutêe lors de la correction des épreuves. Une expérience réalisẻe à l'Institut d'Optique d'Orsay (A. Aspect, Ph. Grangier et G. Roger, 1981, à paraitre) a donné : $\Delta \exp =0,307 \pm 0,004$ en parfait accord avec la prédiction : $\Delta_{\mathrm{M} . Q .}=0,308 \pm 0,002$ 
2.3. Aututres_expériences_avec_photons_de bassse_énergrgie_- 2.3.1. Expérience de Clauser et Freedman (1972). - C'est la première de la série, faite sur une cascade du calcium. Elle a nécessité 200 heures d'enregistrement, et a donné:

$\Delta_{\text {exp }}=0,300 \pm 0,008$

en net désaccord avec l'inégalité B.C.H.H.S. et un parfait accord avec la prédiction quantique prenant en compte les imperfections du montage. 2.3.2. Expérience de Holt et Pipkin (1973).- Réalisée sur une cascade du mercure (différente de celle de Fry), elle a donné:

$\Delta_{\text {exp }}=0,216 \pm 0,013$

en accord avec l'inégalité B.C.H.H.S. et en désaccord avec la prédiction M. $Q$. (0,266 compte-tenu des imperfections).

Aucune raison indiscutable n'a pu être mise en évidence qui pourrait expliquer ce désaccord entre les résultats et la prédiction de la mécanique quantique, mais des hypothèses vraisemblables ont êtê émises par clauser.

2.3.3. Expérience de clauser (1976). - Réalisée sur la même cascade du mercure que Holt et Pipkin, avec quelques variantes expérimentales, elle donne un résultat en accord avec la mécanique quantique, violant les inégalitếs B.C.H.H.S.

2.4. Expériences_avec_photons_y.- 2.4.1. Principe.- Un positron et un électron dans un état singulet s'annihilent, créant une paire de photons $\gamma$. Des arguments similaires à ceux employés pour des photons de basse énergie montrent qu'on a la possibilité d'une expérience "sensible", avec les photons de haute énergie $(0,5 \mathrm{MeV})$ émis.

L'avantage des photons $\gamma$ ainsi émis par paires est qu'ils sont émis dans des directions opposées, et que les détecteurs ont une excellente efficacité.

Malheureusement, il n'existe pas de polariseur permettant une mesure dichotomique pour de tels photons. Pour "mesurer la polarisation", on fait une diffusion (Compton) de ces photons, et on étudie la statistique de diffusion (c'est-à-dire qu'on mesure la probabilité d'être diffusê dans les diverses directions). Puis, par un calcul utilisant la mécanique quantique, on remonte à la polarisation. Il a donc été avancé qu'il y a une inconsistance logique à opérer ainsi. Il y a tout au moins une hypothèse supplémentaire très forte pour pouvoir établir une inégalité de Bell testable expérimentalement.

2.4.2. Expérience de Kasday-Ullmann et $w u$ (1975). - Résultat en accora avec la mécanique quantique, violant l'inégalité de Bell généralisée. 2.4.3. Expérience de Faraci, Gutkowski, Nottarigo, et Pennisi (1974)..Le rêsultat est ici en désaccord avec la mécanique quantique, à la li- 
mite de l'inégalité de Bell. Cette expérience a été remontée et est à nouveau en cours. On attend les résultats.

Ajoutons qu'il y a eu quelques contestations réciproques entre les équipes américaine et italienne sur la façon d'interpréter la statistique de diffusion Compton (de nombreuses corrections doivent être faites, et il y a un lourd traitement informatique pour obtenir un résultat exploitable).

2.4.4. Autres expériences.- D'autres expériences similaires ont été réalisées depuis (I owe et al. à Londres, Bruno et al. à Bologne, Mesenheimer à Freiburg). Elles confirment les prédictions quantiques.

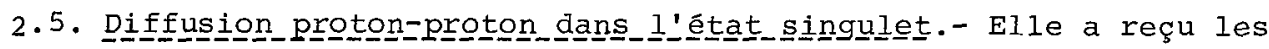
mêmescritiques que les expériences sur les photons $\gamma$, car, ici encore, les polarisations sont calculées à partir de mesures de diffusion.

L'expérience a été faite par Lamehi-Rachi et Mittig (1976) à Saclay. Elle a donné un excellent accord avec la mécanique quantique, et viole une inégalité de Bell génëralisêe faisant appel à de fortes hypothèses supplémentaires. Soulignons que c'est la seule expérience utilisant des particules autres que des photons.

2.6. Conclusion_sur_les_expériences_réalișées.- La majorité des expériences réalisées à ce jour à donné des résultats en accord avec la mécanique quantique. Aucune n'a violé les inégalités de Bell prises au sens strict, mais la majorité viole des inégalités obtenues au prix de quelques hypothèses supplémentaires plus ou moins fortes.

Dans le domaine des photons visibles où les hypothèses supplémentaires nécessaires sont le moins fortes, il $y$ a clairement violation des inégalitês de Bell. De plus, l'accord exact avec les prédictions de la mécanique quantique (qui prennent en compte les imperfections des divers systèmes) est à souligner.

Notons enfin que toutes les causes de perturbation que l'on peut envisager conduisent, lorsqu'on calcule leurs conséquences (par la mécanique quantique), à un affaiblissement systématique des corrélations, et donc à une violation moindre ou à une non-violation des inégalités testées.

3. Expérience avec polariseurs variables.- 3.1 L'hypothèse_de_logalititê et_la_relativivié.- on a vu que pour arriver aux inégalités de Bell, il faut deux hypothèses:

- des corrélations à distance s'expliquent par des causes dans le passé commun des objets sur lesquels portent les mesures;

- hypothèse de localité.

Les expériences semblent en contradiction avec les conséquences de ces hypothèses, dont l'une au moins est donc fausse.

Examinons avèc plus a'attention l'hypothèse de localité (dans le 
cas particulier des paires de photons dont on mesure les polarisations): - la réponse d'un polariseur à un photon ne dépend pas de l'orientation de l'autre polariseur éloigné;

- la façon dont les paires de photons sont émises par la source ne dépent pas des orientations des polariseurs.

Cette double hypothèse semble tout-à-fait raisonnable et conforme à l'image que les physiciens ont du fonctionnement des polariseurs. On peut néanmoins remarquer que cette hypothèse $n$ 'est la conséquence d'aucune loi fondamentale de la physique, et que rien n'interdit d'imaginer 1'existence d'une telle dépendance. On pourrait même interpréter dans ce sens la demande de Bohr de considerer l'ensemble système mesuré/appareil de mesuxe comme un tout non séparable (cł. l'exposé de F. Laloë). Or, si on renonce à cette hypothèse de localitê, les expériences faites à ce jour ne tranchent plus, puisque les inégalités de Bell ne tiennent plus.

Par contre, si on était capable de choisir l'orientation des polariseurs seulement après le départ des photons de la source, I'hypothèse de localité deviendrait en fait la conséquence d'une loi qui semble beaucoup plus fondamentale, le "principe de séparabilité d'Einstein" $\left({ }^{3}\right)$,que l'on peut énoncer dans ce cas particulier: le "réglage" d'un appareil de mesure à un certain instant (évènement A) ne peut pas influencer le résultat d'une mesure effectuée ailieurs à un autre instant (évènement B), si les évènements A et B sont séparés par un intervalle "du genre espace" (un éventuel signal se propageant de A à B devrait aller plus vite que la lumière).

Ainsi, si l'on était capable de changer aléatoirement les orientations a et $b$ avec des temps caractéristiques petits devant le temps que met la lumière pour aller d'un polariseur à l'autre, on aurait pour cette situation expérimentale:

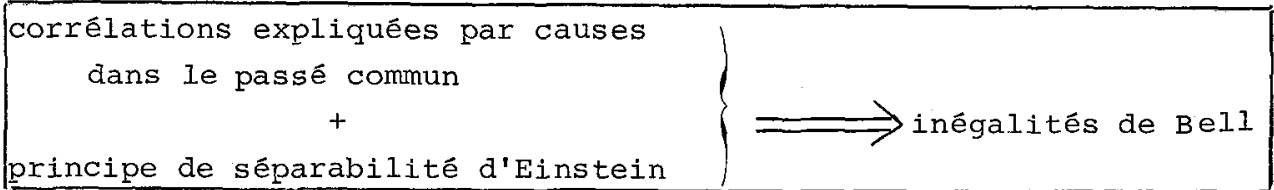

$\left({ }^{3}\right)$ Le "principe de séparabilité d'Einstein" auquel nous faisons référence (cf. exposé de B. d'Espagnat) contient plus de choses que la notion de séparabilité discutée par F. Laloë. Ce principe nous conduit d'une part à admettre qu'il est "possible de distinguer dans un système physique des sous-systèmes possédant une réalité physique intrinsèque" (séparabilité au sens de Laloë); de plus, si ces sous-systèmes sont séparés par un intervalle "du genre espace", une action sur I'un des sous-systèmes ne peut modifier la réalité physique de 1'autre (et donc le résultat d'une mesure effectuée sur ce dernier). Cette dernière notion ("causalité microscopique") joue dans l'espace temos le même rôle que la localité dans l'espace ordinaire, mais il semble à priori beaucoup plus difficile d'y renoncer. 
Or, la mécanique quantique continue de prévoir une violation des inégalités de Bell dans cette situation. Il y a donc de nouveau un conflit que 1 'on peut trancher expérimentalement.

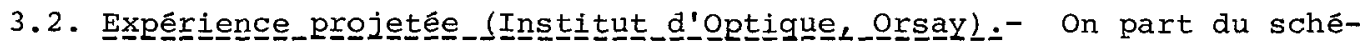
ma C.H.H.S. : photons visibles et polariseurs avec une seule voie de détection (Fig.5). Pour réaliser une modification rapide de l'orientation d'un polariseur, on utilise en fait un commutateur capable d'envoyer la lumière alternativement sur un polariseur, puis sur un autre (Fig.8).

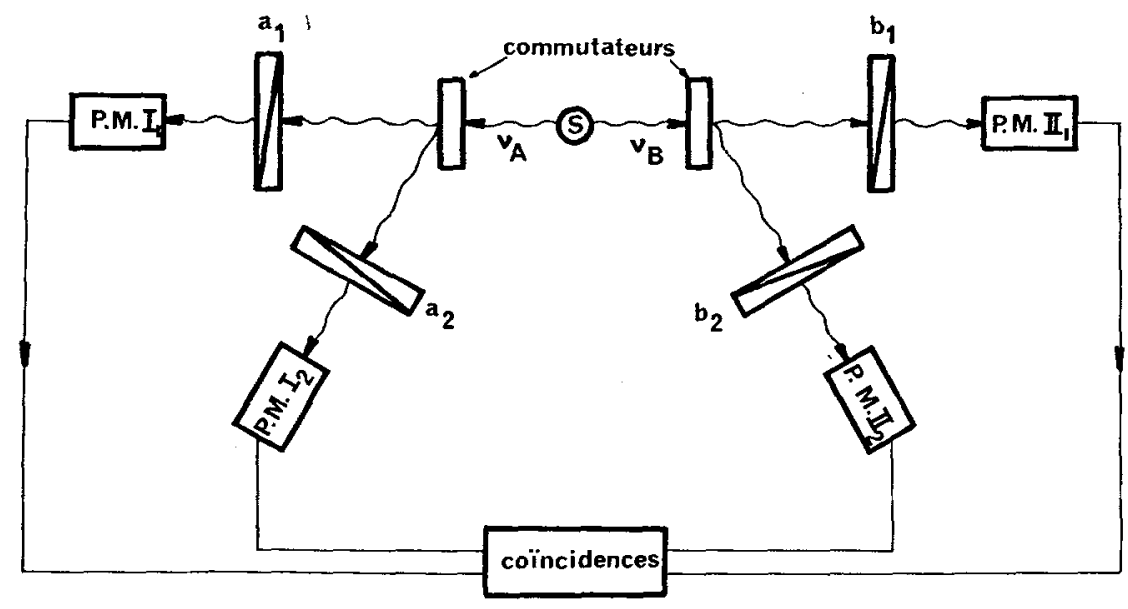

Fig.8.- Expérience en cours.

Ce schéma expérimental peut se ramener à celui de la figure 5, mais où l'on aurait la possibilitê de changer l'orientation de chaque polariseur en un temps plus court que le "temps de vol" des photons.

Tout se passe comme si on avait un seul polariseur I dont l'orientation passe alternativement de $\mathrm{a}_{1}$ à $\mathrm{a}_{2}$, et un polariseur II passant de $\mathrm{b}_{1}$ à $\mathrm{b}_{2}$.

En fait, les commutations ne sont pas vraiment aléatoires, mais néanmoins les deux générateurs qui pilotent les deux commutateurs sont différents, et ils dérivent aléatoirement de façon indépendante. De ce point de vue, on est donc très proche de l'expérience idéale.

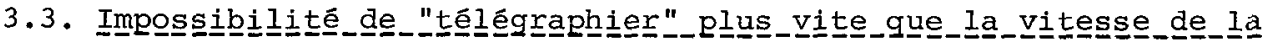

lumière.- Si l'expérience avec polariseurs variables est en accord avec les prévisions quantiques, il faudra en conclure que des corrélations à distance, entre évènements séparés par un intervalle du genre espace, ne peuvent pas s'interpréter simplement en termes de causes communes dans le passé. Le choix de l'orientation d'un polariseur semble donc influer "instantanêment" (c'est-à-dire plus vite qu'à la vitesse 
de la lumière) sur les résultats de mesure à l'autre polariseur. De là à imaginer que l'on puisse "télègraphier" (transmettre des signaux utilisables) à une vitesse supérieure à celle de la lumière, il n'y a qu'un pas ...... qu'il faut bien se garder de franchir.

Il existe une démonstration "technique" rigoureuse de l'impossibilitê de transmettre des signaux utilisables "supralumineux" avec un tel schéma expérimental. On peut comprendre simplement l'essence de cette impossibilité en analysant l'expérience de pensée suivante: on considère le schéma expérimental où des paires de photons corrélés sont analysées par des polariseurs I et II, distants de L (Fig.1), suivis de détecteurs supposés parfaits. On imagine de plus que derrière chaque polariseur, il y a un observateur : $0_{1}$ derrière I et $0_{2}$ derrière II; chaque observateur prend immédiatement connaissance du résultat de sa mesure ( $I_{+}$ou $I_{-}$pour $0_{1^{\prime}}$ II $I_{+}$ou II_ pour $0_{2}$ ); de plus, chaque observateur est capable de modifier très rapidement, à un moment quelconque, l'orientation de son polariseur.

Supposons que $\mathrm{O}_{2}$ change l'orientation du polariseur II, $\mathrm{O}_{1}$ peutil instantanément en être informé? La mécanique quantique prédit que, dans tous les cas, chaque observateur voit 50\% de détection + , et $50 \%$ de détection. Ces taux n'êtant pas modifiés, $O_{1}$ ne peut, avec des comptages simples, rien connaitre de l'orientation b. Par contre, la mécanique quantique prédit que les corrëlations (par exemple $P_{++}(a, b)$ ) sont instantanément modifiẻes. Mais pour connaitre $P_{++}(a, b)$ a l'instant $t$, l'observateur $0_{1}$ doit prenare connaissance des résultats de mesure en II à l'instant $t$; et il pourra recevoir une telle information, communiquée par exemple par radio, au plus tôt à l'instant $t+\frac{L}{C}(C=$ vitesse de la lumière). Ce n'est donc qu'à $t+\frac{I}{\bar{C}}$ qu'il saura que $P++$ $(a, b)$ avait changé à $I^{\prime}$ instant $t$, et donc que $\mathrm{O}_{2}$ avait tourné son polariseur à l'instant $t$; cette information aura donc voyagé à la vitesse de la lumière.

En conclusion, si cette expérience donne un résultat conforme aux prédictions de la mécanique quantique, elle soulignera un des points les plus étonnants du problème et renforcera sans doute la conviction de ceux qui pensent qu'il faut renoncer à la séparabilitê. Néanmoins, ce résultat ne pourrait être considéré comme une violation de la relativité, au moins sous sa forme opérationnelle (impossibilité d'envoyer un signal utilisable plus vite que la lumière). 
Discussion après l'exposé de : A. Aspect

Intervention de : A. Shimony

Dr Aspect pointed out that in the photon polarization experiments some auxiliary hypotheses (which unfortunately are untestable) had to be made in ordex to derive from Bell's locality assumption an inequality concerning coincidence counting rates. The source of the trouble is the inefficiency of the photo-detectors in the frequency range where the polarization analyzers are efficient. Clauser and Horne adapted a model of Bell to show that quantum mechanical counting rates could be produced by apparatus which satisfies the locality assumption, provided that the photo-detectors are sufficiently low in efficiency. Hence, there is a serious loophole in the present experimental disconfirmation of local hiddenvariables theories. In order to block this loophole, T.K. Lo and I have designed an experiment which uses sodium molecules. By proper laser excitation (actually, induced Raman scattering) the molecule is dissociated into 2 atoms which have a total singlet spin state. It seems that stern-Gerlach analysis of the spins and hot wire detection of the atoms will be sufficiently efficient to block the clauser-Horne model and to yield counting rates which will directly test Bell's inequalities without auxiliary hypotheses. (See T.K. Lo and A. Shimony, to appear in Phys. Rev. A, 1981).

\section{Intervention de_: M. Paty}

D'Espagnat vient de dire - et il l'a, je crois, écrit également dans son livre - que "c'est la même chose de dire qu'un système est inséparable que de dire qu'il y a transmission instantanêe à distance". J'avoue qu'une telle formulation me choque profondément. En effet j'y vois tout d'abord une incohêrence logique. Le premier membre de l'identification se réfère à un "système inséparable", c'est-à-dire inséparable d'une maniẽre générale, donc quelle que soit la représentation que l'on se donne de ce système - et, en particuliex, inséparable même dans une représentation spatiale, en termes de caractérisation locale. Le second membre porte sur une "transmission instantanée à distance" d'une influence ou d'une interaction. Or, parler de transmission à distance, cela signifie qu'on distingue spatialement les systèmes en question, qu'on les sépare : ce que le premier membre de la phrase déclarait impossible par définition. 
Cette formulation me choque également parce qu'elle essaie de résoudre l'incompatibilité de deux exigences, de deux approches, qui me semble, comme je viens de le dire, d'ordre avant tout logique, non-sêparabilité d'une part, sêparation locale de l'autre - par l'appel à quelque chose qui est d'ordre physique : la transmission à distance, qu'elle soit influence ou interaction. Or cette notion, ici, est parfaitement ad hoc, choisie sur mesure pour pallier une difficulté logique - mais qui n'est pas vue comme telle. Elle ne se présente pas comme quelque chose de vraiment physique : "instantanée à distance" contredit la relativité restreinte, et cette contradiction me parait suffisamment importante pour demander une véritable observation. Certains prétendent que cette violation de la relativité est rêellement, bien qu'indirectement, vérifiée dans ce type de processus. Je ne crois pas que ce soit exact : ce ne serait vrai que si les concepts en jeu correspondaient eux-mêmesà des éléments physiques observés ; or ces concepts sont flous, ambigus, contradictoires, puisqu'on ne sait pas ce qu'on dit exactement quand on parle de ces sous-systèmes de l'état final - ils ne sont pas caractérisés par la mécanique quantique. Comment alors parler de vérification, ou d'observation, d'un signal quelqu'il soit entre des entités sans spécification, ou du moins spécifiées d'une manière très lâche?

Bohm, dans son article de 1952, supposait la transmission "instantanée de perturbations incontrôlables d'une particule à l'autre". En fait de telles "perturbations" ou "influences" sont une traduction dans le langage - locale d'un formalisme non-local, et cette traduction n'est pas sans inconvénients. On pourrait considérer, avec J.P. Vigier, qu'il s'agit d'influence "non-physiçue", puisqu'il n'y a pas d'échange d'énergie. Mais qu'est-ce qu'un échange d'information sans échange d'énergie? Iaut-il entenare cette explication comme "purement mathématique", sans contrepartie physique? Un tel recours à un mécanisme mathématique non-physique - et, ce, a priori - me paraît susceptible de conduire à des confusions, et surtout me semble inutile. Car il n'y a pas d'interaction, ni de transmission de quoi que ce soit s'il n'y a pas caractêrisation de systèmes séparés. Il faut donc, à mon avis, s'en tenir aux concepts impliqués dans la thêorie et dans le formalisme, et tenter d'exprimer la non-séparabilité de cette façon. Si l'on parle d'"influence", ce ne peut être, en quelque sorte, qu'au niveau du vocabulaire et des concepts : c'est sous l'influence d'une approximation en termes de concepts classiques - et non quantiques - qu'on parle des sous-systèmes de l'état final. Ou, pour parler un peu comme Bohr (au moins dans le deuxième sens de sa proposition dont j'ai parlé prêcédemment) qui critiquait le sens de l'expression "sans perturber aucune- 
ment le système", employée par Einstein, Podolski et Rosen, il ne s'agit pas d'une perturbation mécaniq̣ue, mais d'une définition du système dont on parle; ce n'est pas d'une influence physique qu'il est question, mais d'une influence des conditions de l'approche de ce système ( $a u$ sens conceptuel).

Il y aurait beaucoup à dire sur cette question - épistémologiquement fondamentale - des rapports entre la théorie quantique et les concepts classiques. Bohr et Heisenberg pensaient que la thêorie quantique devait, de toute nécessité, être exprimée dans les concep̣ts classiques: d'où nos difficultés. C'est en fait cette question qui me parait être au centre de tous nos problèmes. N'y a-t-il pas des concepts appartenant en propre à la théorie quantique et auxquels il fauarait autant que possible se tenir? La non-séparabilité me parât en être un, le plus typique peut-être. ( Ceci dit indépendament du fait de savoir si la mécanique quantique est formellement satisfaisante. Même si on la considère pas telle, il faut bien, comme le dit excellemment John Bell, "vivre désormais avec la non-séparabilité"). 